\title{
Oxygen PFIB/SEM tomography of Biological Samples
}

\section{Daniela Slamková}

Thermo Fisher Scientific, Brno, Jihomoravsky kraj, Czech Republic

Corresponding author: daniela.slamkova@thermofisher.com

Over the past years, the Focused Ion Beam Scanning Electron Microscopy (FIB/SEM) system has been established as a key technology in life science and has become a common method for 3D analysis of biological samples at high isotropic resolution. A limiting aspect of this technique is the consideration of suitable sample preparation and resin type. Currently, conventional FIB/SEM sample preparation requires enhanced contrasting and embedding in epoxide resins like Epon or Durcupan. These hydrophobic resins are commonly employed for ultrastructural studies, as they are less prone to milling artifacts in galliumbased FIB/SEM systems. In contrast, Acrylic resins (LR White, Lowicryl) tend to generate severe milling artifacts and have low stability under the galium FIB milling (Figure 1B). As a result, the use of these resins for FIB/SEM tomography is very limited, although a range of life science studies will benefit from the possibility to use Acrylic resins for adequate preservation of the cellular structure, as well as the preservation of a fluorescent signal. Here, we present a PFIB-SEM system that can address some of these limitations. Helios ${ }^{\mathrm{TM}} 5$ Hydra DualBeam is a system featuring Inductively Coupled Plasma (ICP) with four ion species (Xe, Ar, O, N) as an ion source. Special focus is given on the oxygen beam, which provides enhanced sputter rates and extends the FIB-SEM 3D volume acquisition to samples embedded in acrylic resin (Figure 1E). In contrast to gallium FIB, oxygen PFIB displays superior resin compatibility with most resins used in life science and produces a smooth block face without beam-induced damage (Figure 1C) resulting in an improved SEM contrast. We demonstrate that PFIB technology allows for more flexible sample preparation and enables researchers to choose the preparation method that serves best the scientific question. Next to extending compatibility with multiple resins commonly used in biology the Helios Hydra PFIB also offers new opportunities for exploring 3D visualization and accessing large volumes. The Spin Mill technique is a method for polishing horizontal planes of the sample surface and thus can provide a Serial Block-face Electron Microscopy (SBEM) approach using the capability of the focused ion beam. This technique is based on removing a thin layer from the resin-embedded sample at a glancing angle (typically $4^{\circ}$ to the sample surface, Figure 2A) using the PFIB ion beam (for biological resin-embedded samples typically oxygen) and subsequent imaging of the newly created sample surface by the electron beam. Very thin slices in the nm range can be automatically milled from a large region (up to $1 \mathrm{~mm} 2$ ) and imaged repetitively. The Spin Mill technique allows for multiple sites of image acquisition in one experiment, generating a high-resolution 2D image stack for subsequent 3D visualization of features of interest at nanoscale resolution (Figure 2E). The availability of oxygen PFIB/SEM system represented by Helios ${ }^{\mathrm{TM}} 5$ Hydra DualBeam extends the potential of FIB/SEM tomography applications in life-science research. It is a powerful instrument for imaging a wide range of biological samples at a nanoscale resolution. In addition, the capability of delivering an order of magnitude higher currents (up to $2.5 \mu \mathrm{A}$ ) enhances the acquisition speed of PFIB-SEM resulting in increased throughput and reliable reconstruction of the sample volume in three dimensions. 

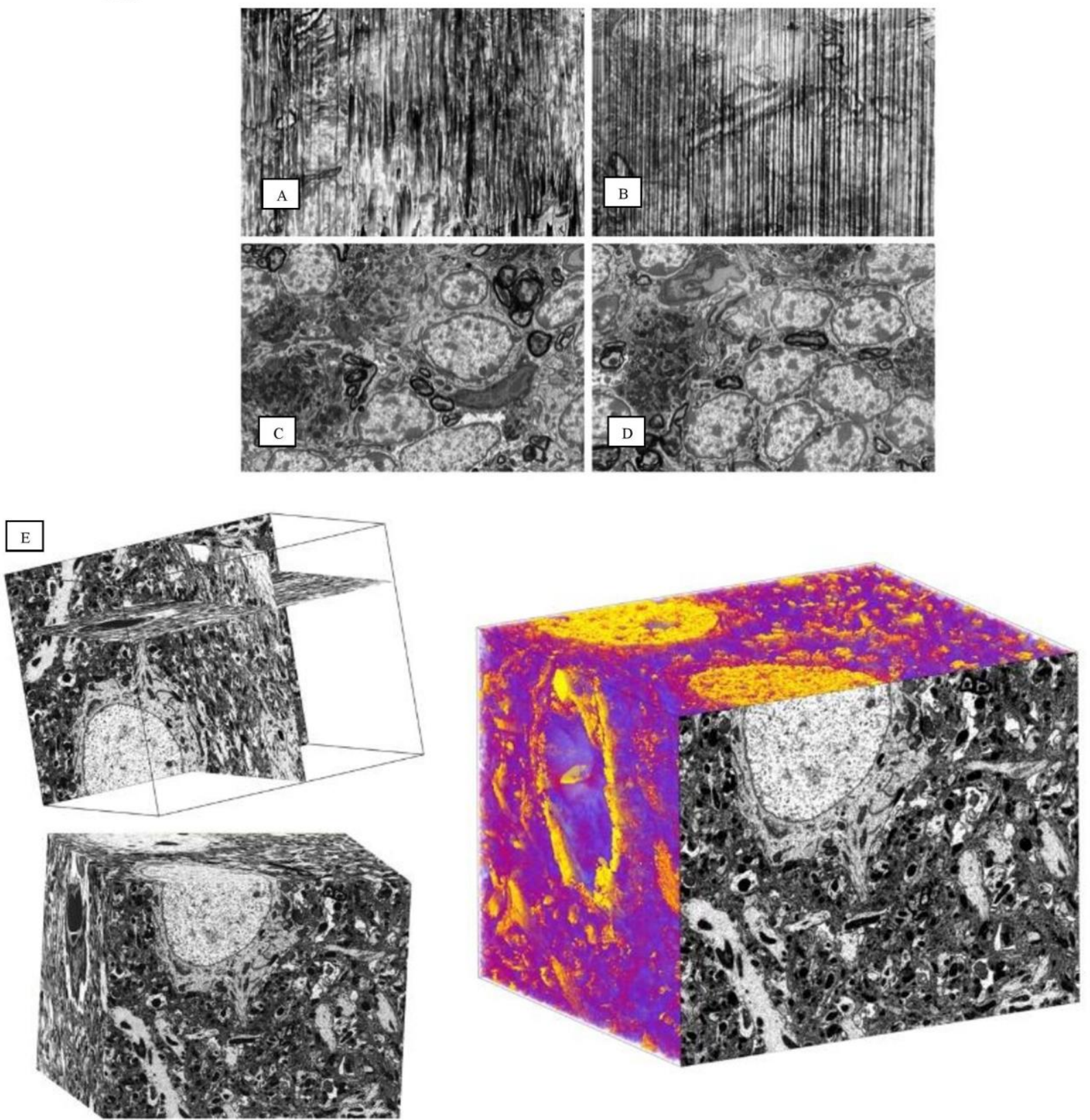

Figure 1. Figure 1. Mouse brain tissue embedded in LR White resin, chemically fixed, milled with (A) Xenon PFIB: 30 kV, 60 nA. (B) Gallium FIB: 30 kV, 65 nA. (C) Oxygen PFIB: 30 kV, 45 nA. (D) Nitrogen PFIB: $30 \mathrm{kV}, 23 \mathrm{nA}$. ICD detector( $2 \mathrm{kV}$; $200 \mathrm{pA}$ ), pixel width is $9 \mathrm{~nm}$, HFW is 28 um. (E) 3D reconstruction of a mouse brain tissue embedded in acrylic resin. Milling parameters: oxygen; 0,61 nA; $30 \mathrm{kV}$. Volume is (X x Y x Z) 23,4 $\mu \mathrm{m} \times 18,2 \mu \mathrm{m} \times 20 \mu \mathrm{m}$. Slice thickness is $10 \mathrm{~nm}$. Total number of slices is 2000 . 

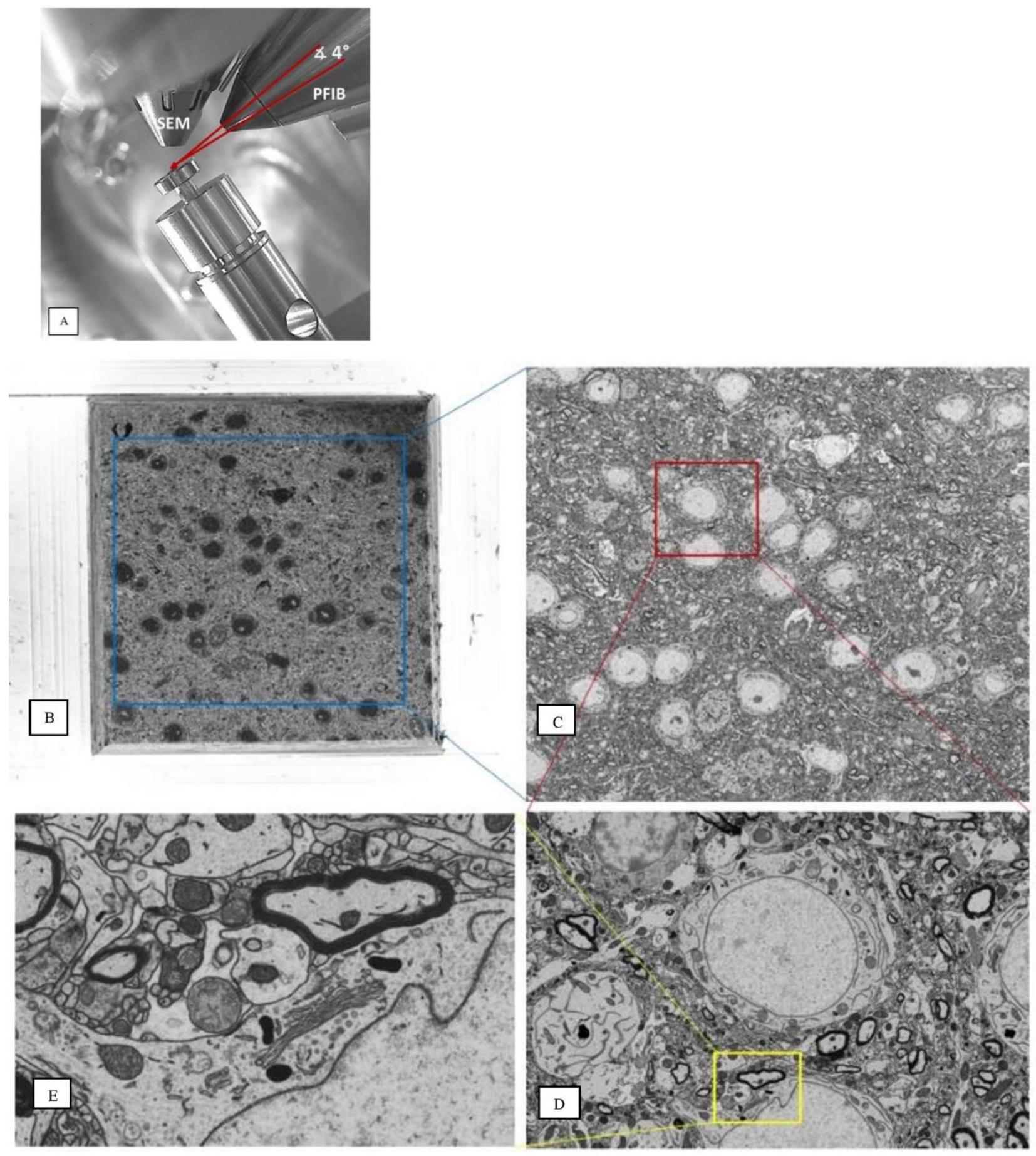

Figure 2. Figure 2. Spin Mill technique. (A) The stage is tilted to the glancing angle $4^{\circ}$ to the PFIB beam and continuously rotated by a defined number of steps, at which is the sample surface exposed to PFIB. After milling process, the stage is automatically tilted, so that the electron beam is perpendicular to the sample surface from which SEM images are taken. (B) Perpendicular electron view of the sample, the whole top-face (189 x $187 \mathrm{um}$ ) is milled during Spin Mill procedure. (C) First imaging area, HFW is 147 um. (D) Second imaging area, detail of a neuron cell, HFW is 54 um. (E) Third imaging area, subcellular detail, HFW is 8 um. 